\title{
How miRNAs can protect humans from coronaviruses COVID-19, SARS-CoV, and MERS-CoV
}

\author{
Anatoliy Timofeevich Ivashchenko \\ Al-Farabi Kazakh National University \\ Almaty, Kazakhstan \\ a.iavashchenko@gmail.com
}

\author{
Aizhan Kazievna Rakhmetullina \\ Al-Farabi Kazakh National University \\ Almaty, Kazakhstan \\ zhanullina1994@gmail.com \\ Dana Evgenievna Aisina \\ Al-Farabi Kazakh National University \\ Almaty, Kazakhstan \\ dana.aisina03@gmail.com
}

\author{
Aigul Nurlankyzy Akimniyazova \\ Al-Farabi Kazakh National University \\ Almaty, Kazakhstan \\ akimniyazova@gmail.com
}

\begin{abstract}
Viral diseases cause significant harm to human health and often cause high mortality. In the past twenty years, humanity has undergone infection by SARS-CoV (severe acute respiratory syndrome), MERS-CoV (Middle East respiratory syndrome) and COVID-19 coronaviruses, which spread from animals to humans and from person to person. These diseases have led to large economic losses. To fight coronaviruses and other viruses, it is proposed to use miRNAs, which regulate protein synthesis at the translational level. Out of 2565 miRNAs, miR-4778-3p, miR-6864-5p and miR-5197-3p were identified as the most effectively interacting with the gRNA of SARS-CoV, MERS-CoV and COVID-19, respectively. Based on the miR4778-3p, miR-6864-5p and miR-5197-3p sequences, complete complementary miRNA (cc-miR) binding sites in the gRNA coronaviruses were created. Detected binding sites of these ccmiRs did not form intramolecular complexes in the 2D structure of the gRNA of SARS-CoV, MERS-CoV, and COVID-19 with a value of more than $85 \%$. Therefore, cc-miRs will bind gRNA at these sites without competition. The cc-miRs for SARS-CoV, MERS-CoV, and COVID-19 did not have target genes among the 17508 human coding genes with a $\Delta G / \Delta G m$ of more than $85 \%$, which implies the absence of side effects of cc-miRs on the translation of human mRNAs. cc-miRs can be used as therapeutic agents by incorporating them into exosomes or other vesicles and introducing them into the blood or lung by inhalation. The introduction of cc-miR into the blood will suppress the reproduction of the virus in the blood and in all organs into which it can enter.
\end{abstract}

Keywords - SARS-CoV, MERS-CoV, COVID-19, virus, $m R N A, m i R N A$, disease, treatment

\section{Introduction}

Viral diseases are difficult to predict, which requires prompt responses to their occurrence. Unfortunately, at present, specific drugs do not exist for all viruses. Coronaviruses COVID-19, SARS-CoV (severe acute respiratory syndrome) and MERS-CoV (Middle East respiratory syndrome) are highly pathogenic, and currently, there are no effective drugs to treat them [1]. Therefore, new approaches to the treatment of viral diseases are required. All viruses multiply using a nucleic acid synthesis system and a protein synthesis system in an infected cell. In animal cells, protein synthesis is controlled by miRNA (mRNA-inhibiting RNA). These nanoscale molecules specifically interact with the mRNAs of target genes and can reversibly suppress translation or contribute to the degradation of mRNA. This biological role of miRNAs can help in the fight against viral reproduction, since the synthesis of viral proteins occurs in the host cell. The use of miRNAs for this purpose requires with the fulfillment of a number of conditions. The miRNA must highly specifically bind to the mRNA of the viral genome (gRNA) or parts of its genome, which can be translated as a whole or in fragments. An arbitrary set of nucleotides in synthetic miRNAs that bind with complete complementarity to the RNA genomes of viruses can have many side effects consisting of the binding of these miRNAs to human mRNAs. To identify target miRNAs of genes, a program that adequately evaluates the interaction of this miRNA with all genes of the human genome is required. The MirTarget program we created successfully determines the characteristics of the interaction of known miRNAs with any RNA nucleotide sequences. Using this method, the effect of known human miRNAs on the mRNA of plant and animal genes was evaluated. It is currently relevant to identify human miRNAs that can affect the expression of the COVID-19, SARS-CoV and MERS-CoV coronavirus genomes at the translation stage. It is necessary to select miRNAs that can most effectively bind to the nucleotide sequence of the RNA genomes of these coronaviruses. The aim of this work was to construct miRNAs specific for coronaviruses COVID-19, SARS-CoV, and MERS-CoV that will not affect the expression of human or animal genes.

\section{Materials and Metods}

The nucleotide sequences of 2565 miRNAs were downloaded from miRBase (http://mirbase.org, release 22.1). The nucleotide sequences of genes and the coronavirus genome COVID-19, MERS-CoV, SARS-CoV were obtained from NCBI (http://www.ncbi.nlm.nih.gov). A search for the target genes of miRNAs was performed using the MirTarget program [2]. The MirTarget program works with the miRNA and mRNA nucleotide sequences in a special format. This program determines the following binding characteristics: the start of the miRNA binding site on the mRNA; the location of the miRNA binding site (3'UTR, 5'UTR, CDS); the interaction free energy $(\Delta \mathrm{G}, \mathrm{kJ} / \mathrm{mole})$; and the nucleotide interaction schemes between miRNAs and mRNAs. The ratio of $\Delta \mathrm{G} / \Delta \mathrm{Gm}(\%)$ was determined for each binding site, where $\Delta \mathrm{Gm}$ is equal to the free energy binding of miRNA with its full complementary nucleotide sequence. The MirTarget program found hydrogen bonds between adenine (A) and uracil $(\mathrm{U})$, guanine $(\mathrm{G})$ and cytosine $(\mathrm{C}), \mathrm{G}$ and $\mathrm{U}, \mathrm{A}$ and $\mathrm{C}$. The distances between $\mathrm{A}$ and $\mathrm{C}$ were equal to 1.04 nanometers, between $\mathrm{G}$ and $\mathrm{C}$, and between $\mathrm{A}$ and $\mathrm{U}$ were equal to 1.03 nanometers, and between $\mathrm{G}$ and $\mathrm{U}$ equal to 1.02 nanometers. The numbers of hydrogen bonds in the G-C, A- 
$\mathrm{U}, \mathrm{G}-\mathrm{U}$ and A-C interactions were found to be 3, 2, 1 and 1, respectively.

\section{Results and Discussion}

To compile the nucleotide sequence of a fully complementary miRNA (cc-miR) to its binding site in the gRNA of COVID-19, it was necessary to find the cc-miR precursor. Despite the large size of the COVID-19 genome, in comparison with the human protein coding genes, only a few human miRNAs could bind to the viral genome with a $\Delta \mathrm{G} / \Delta \mathrm{Gm}$ value of $89 \%$. We chose this value as a criterion for the adequacy of determining binding sites based on the requirement that different miRNAs with a length of $22 \mathrm{nt}$ differ in two or more nucleotides, which allows them to act specifically. A decrease in this criterion, for example, by $5 \%$, leads to an exponential increase in the number of putative target genes of a particular miRNA, which results in an uncertainty in the selectivity to the target genes of this miRNA. To create cc-miR, we selected miR-5197-3p, which out of the 2565 known miRNAs (miRBase), binds to the gRNA of the COVID-19 virus with the largest $\Delta \mathrm{G} / \Delta \mathrm{Gm}$ value of $89 \%$ and a miRNA length of $23 \mathrm{nt}$.

Of the 17,508 human genes available in our gene database, miR-5197-3p bound to the mRNA of only a few genes with similar characteristics. Therefore, the use of miR-5197-3p as a therapeutic agent will cause side effects on the identified genes.

Based on this scheme, a structure for cc-miR2 (cc-miR for the gRNA of COVID-19) with a length of $25 \mathrm{nt}$ was proposed with the replacement of non-canonical nucleotide pairs by canonical ones and the addition of $\mathrm{G}$ and $\mathrm{U}$ nucleotides at the ends of miR-5197-3p to increase the free interaction energy of cc-miR2 with the gRNA of COVID-19. The miRNAs with a length of $25 \mathrm{nt}$ are found among natural miRNAs (miRBase) and can interact with gRNA as part of RISC (RNA-induced silencing complex). The created cc-miR2 was completely complementary to the gRNA and interacted weakly with mRNA of 17508 genes. This result gives confidence that ccmiR2 can interact with gRNA without side effects on the human protein-coding genes.

Similarly, cc-miRs were designed to bind to the MERS$\mathrm{CoV}$ (cc-miRm) and SARS-CoV (cc-miRs) genomes. Of the 2565 known human miRNAs, miR-6864-5p and miR-4778$5 \mathrm{p}$ could interact more strongly than other miRNAs with the gRNA of MERS-CoV and SARS-CoV, respectively. The ccmiRm and cc-miRs do not bind to mRNAs of the 17,508 studied human genes with $\Delta \mathrm{G} / \Delta \mathrm{Gm}$ values above $85 \%$.

Therefore, these synthetic miRNAs are not expected to have any side effects upon interacting with human genes. The nucleotides of the cc-miR2, cc-miRm, cc-miRs binding sites during intramolecular interactions in the COVID-19, SARS$\mathrm{CoV}$, and MERS-CoV genomes can bind to other gRNA sites with a value of $\Delta \mathrm{G} / \Delta \mathrm{Gm}$ less than $71 \%$. Consequently, synthetic cc-miR2, cc-miRm, and cc-miRs will interact with the binding sites in the genomes of the three coronaviruses without competition with other gRNA sites.

Generating cc-miR is an inexpensive and relatively short procedure, similar to the synthesis of primers. In cell culture, the effects of cc-miR and miRNA on coronaviruses need to be tested. The proposed hypothesis can be confirmed in the laboratory with the approval and ability to conduct inexpensive and time-saving tests of the proposed cc-miR as a means of combating coronavirus. Since the size of cc-miR is approximately $10 \mathrm{~nm}$, it can be delivered with blood to many organs as a component of ordinary exosomes in human blood, which measure 30-60 $\mathrm{nm}$. The cc-miR contained in exosomes can be introduced into the lungs by inhalation. The proposed method for combating coronaviruses using miRNA does not have side effects and is economically inexpensive. Like all human miRNAs, cc-miR is susceptible to degradation by nucleases, and its removal from the body is not difficult.

A well-known observation that COVID-19 infects children under the age of 15 less than adults over 60 can be explained by the expression of piwi-interacting RNA (piRNA) and miRNA, whose concentrations change during ontogenesis: the proportion of pi-RNA decreases with age, and the proportion of miRNA increases. It is possible that the expression of one or more pi-RNAs that can bind to the viral gRNA decreases during ontogenesis, and the virus begins to multiply. Similarly, the concentration of miRNAs capable of binding to the gRNA and are expressed in the early stages of ontogenesis can decrease, and the virus begins to multiply.

\section{Conclusion}

The results show that it is not difficult to select the nucleotide composition of miRNA, siRNA or pi-RNA for target binding to mRNA. The challenge is to predict and verify the absence of side effects of the small RNA on other genes. Unfortunately, this mandatory analysis is performed only at the stage of experimental testing, which will take a long time and is not guaranteed to end successfully. Our proposed method of searching for synthetic miRNA and evaluating its side effects can significantly simplify the generation of the miRNA of interest.

\section{ACKNOWLEDGMENT}

We thank Pyrkova A. Yu for the help in the data analysis and interpretation.

\section{REFERENCES}

[1] K. Alburikan, H. Abuelizz, "Identifying factors and target preventive therapies for Middle East Respiratory Syndrome sucsibtable patients," Saudi Pharm J, vol. 28, pp. 161-164, 2020. DOI: 10.1016/j.jsps.2019.11.016.

[2] A. T. Ivashchenko, O. A. Berillo, A. Y. Pyrkova, R. E. Niyazova, S. A. Atambayeva, "MiR-3960 Binding Sites With mRNA of Human Genes," Bioinformation, Vol. 10, pp. 423-427. 2014. DOI: $10.6026 / 97320630010423$. 